\title{
Feynman e os pólarons
}

\author{
Feynman and the polarons
}

\author{
Nelson Studart ${ }^{*}+10$
}

${ }^{1}$ Universidade Federal do ABC, Centro de Ciências Naturais e Humanas, Santo André, SP, Brasil

\author{
Recebido em 13 de Fevereiro, 2018. Aceito em 15 de Fevereiro, 2018.
}

\begin{abstract}
O estudo de quase-partículas formadas pela interação elétron-fônon em cristais iônicos (os pólarons) despertou o interesse de Feynman pela possibilidade de encontrar alguma conexão com a supercondutividade e por proporcionar uma aplicação direta do seu formalismo da eletrodinâmica quântica. Para calcular a energia do estado fundamental do pólarons, Feynman desenvolveu um método variacional que pode ser aplicado em cálculos de integrais de caminho e que o deixou satisfeito. Nesse trabalho, o artigo original de 1955 de Feynman é analisado com inserções de caráter histórico que contribuem para compreender a sua abordagem na solução de problemas.
\end{abstract}

Palavras-chave: pólarons, integrais de caminho; Feynman; história da física.

The study of quasi-particles formed by the electron-phonon interaction in ionic crystals (the polarons) aroused Feynman's interest in the possibility of finding some connection with superconductivity and for providing a direct application of his formalism of quantum electrodynamics. To calculate the ground state energy of the polaron, Feynman developed a variational method that can be applied to calculations of path integrals and that gave him some pleasure. In this work, Feynman's original 1955 article is analyzed with historical insights that help to understanding his approach to problem solving.

Keywords: polarons, path integrals; Feynman; history of physics.

Após o sucesso de seus trabalhos acerca da formulação da mecânica quântica [1] e da eletrodinâmica quântica [2] e da rápida expansão das técnicas diagramáticas por todo o mundo [3], Feynman se interessa pela pesquisa em sistemas da matéria condensada [4]. Nesse sentido, para o leitor brasileiro, é importante enfatizar que suas ideias sobre a superfluidez do hélio líquido foram desenvolvidas, em parte, nos dez meses da licença sabática [1951-1952] que gozou no CBPF, a convite de Leite Lopes $[5,6]]^{1}$

O objetivo de Feynman era compreender a superfluidez do hélio líquido do ponto de vista microscópico estendendo a teoria fenomenológica de Landau para esse domínio e, com vistas a possíveis conexões com a questão crucial na época que consistia em estabelecer uma teoria microscópica para o fenômeno da supercondutividade em metais. [7] A partir desse interesse pela supercondutividade, Feynman se deparou com um velho problema da década de 30 em que ele percebeu a possibilidade de aplicar seu formalismo da eletrodinâmica quântica para a solução de um caso concreto da física do estado sólido. É interessante conhecer como Feynman se deu conta desse problema.

\footnotetext{
* Professor Sênior da Universidade Federal de São Carlos.

†Endereço de correspondência: n.studart@gmail.com

${ }^{1}$ Em 1953, Feynman esteve novamente no Brasil, participou da SBPC em Belo Horizonte, antes de ir para o Japão participar do Symposium on liquid helium and superconductivity em setembro, quando apresentou o trabalho que veio a ser publicado no Physical Review sendo seu primeiro artigo sobre o tema.
}

Josep T. Devresse [8], com longa militância na pesquisa em pólarons, fez esse depoimento obtido de uma consulta direta a Feynman:

In the early fifties H. Fröhlich gave a seminar at Caltech. In this seminar he discussed the weak-coupling polaron mass as derived by him: $m^{*}=m_{\mathrm{b}} /(1-\alpha / 6)$. He suggested that, if the electron-phonon coupling could be accurately treated for intermediate coupling (in particular around $\alpha \approx 6$ ) this might lead to new insights in the theory of superconductivity (this was before BCS) (Feynman, 1973, private communication). Feynman was among the audience. He went to the library to study one of Fröhlich's papers on polarons (Fröhlich, 1954). There he got the idea to formulate the polaron problem "into the Lagrangian form of quantum mechanics and then eliminate the field oscillators".

No depoimento a seu biógrafo Jagdish Mehra [6], Feynman revela, no seu estilo brincalhão, outro motivo para sua ida à Biblioteca: a beleza das bibliotecárias do Caltech. Em carta de 8 de setembro de 1954, encaminhou a Herbert Fröhlich a solução do desafio, possivelmente feito durante o seminário:

We have very pretty librarians. Last week I went to enjoy the scenery and picked up Ad- 
vances [in] Physics as a cover. I read your article. I became more interested in it than in the librarians and resolved to accept your challenge to find a method uniformly valid over the entire range of [the coupling constant]. Enclosed is my solution... What to have to do to understand superconductivity?

Feynman estava certo. Os resultados obtidos para a energia e massa efetiva do pólarons são válidos, como será visto em seguida, para todo valor da constante de acoplamento e são os melhores comparados aos demais métodos. Contudo, como é bem conhecido, esse insight não funcionou na supercondutividade.

E que são os pólarons?

Lev Landau, em 1933, sugeriu a possibilidade de que um elétron movendo-se livremente em um cristal iônico poderia ser aprisionado devido a sua interação com fônons ópticos da rede.[9] Seu principal interesse estava relacionado com os defeitos gerados na rede, como os centros F de cor em cloreto de sódio, mas não descreve o mecanismo de aprisionamento. Foi Salomon Pekar quem introduziu e cunhou o termo "pólarons" em trabalhos de 1946, definindo-o como um estado de elétron fortemente acoplado à polarização induzida pelo deslocamento de átomos em cristais iônicos.[10] Finalmente em 1948, Landau e Pekar consolidaram o conceito do pólaron como um portador de carga em sólidos admitindo que "o portador de corrente em redes iônicas de cristais é o pólaron ao invés do elétron da banda de condução" (uma quasepartícula na terminologia atual) e calcularam a massa efetiva do pólaron no regime de acoplamento forte. [11] A Fig. 1 é uma representação pictórica do pólaron formado a partir da interação do elétron com a rede deslocada do cristal.

Em 1937, Herbert Fröhlich produziu um estudo quantitativo do espalhamento do elétron em cristais iônicos. Nesse trabalho ele propôs a hoje famosa Hamiltoniana ${ }^{2}$ que leva seu nome [12]

$$
H=\frac{p^{2}}{2 m_{b}}+\sum_{k} \hbar \omega_{L O} a_{k}^{\dagger} a_{k}+\sum_{k}\left(V_{k} a_{k} e^{-i \mathrm{k} . \mathrm{r}}+\text { h.c. }\right)
$$

em que o primeiro termo corresponde ao movimento do elétron de condução de massa $m_{b}$, o segundo é o termo de fônons e o último descreve a interação entre o elétron e o campo de fônons de frequência $\omega_{L O}$ (longitudinal óptico) com

$$
V_{k}=-\frac{\hbar \omega_{L O}}{k}\left(\frac{\hbar}{2 m_{b} \omega_{L O}}\right)^{1 / 4}\left(\frac{4 \pi \alpha}{\Omega}\right)^{1 / 2} .
$$

\footnotetext{
2 Fröhlich em seu trabalho original adota o modelo elástico de Debye para os sólidos e calcula o termo de interação do elétron livre com as ondas de polarização (frequência constante) por meio da equação de Poisson para o potencial elétrico $\phi$ e a lei de Gauss para a polarização $\mathbf{P}, \nabla^{2} \phi=4 \pi \nabla \cdot \mathbf{P}$
}

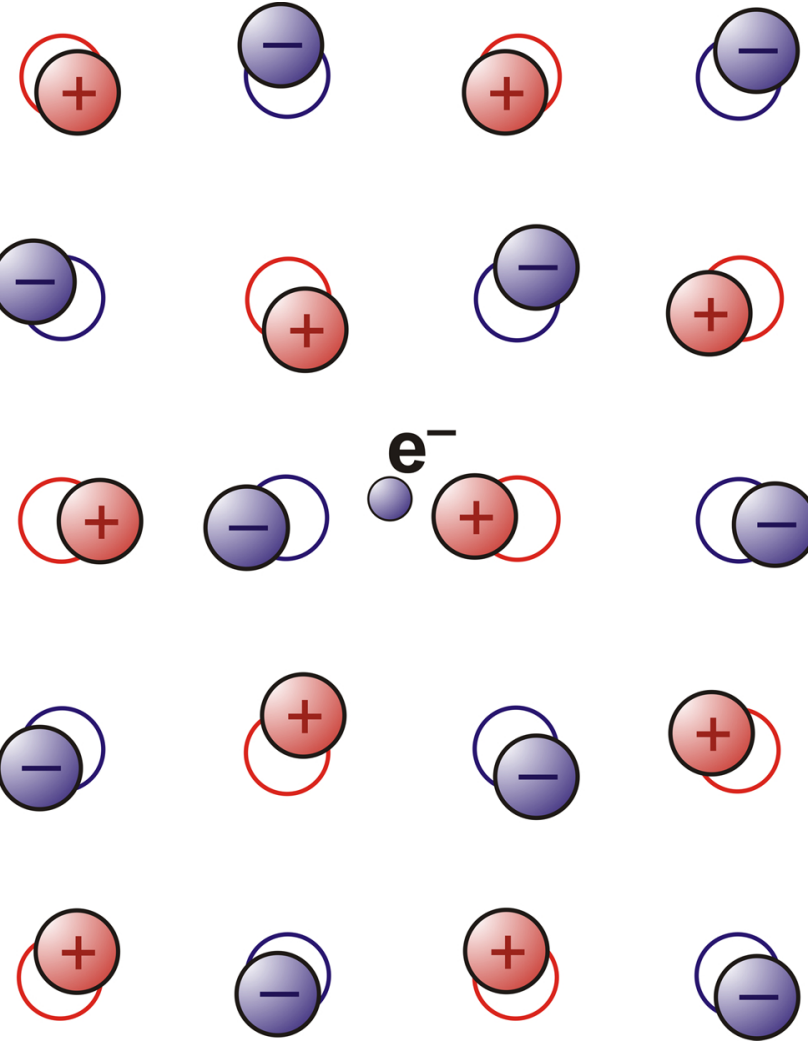

Figura 1: Concepção artística do pólaron: um elétron desloca os átomos ao seu redor produzindo um campo de polarização no cristal. Fonte: Wikipedia: https://goo.gl/rZ2qVi

A constante de acoplamento, introduzida por Fröhlich, é definida como

$$
\alpha=\frac{e^{2}}{\hbar}\left(\frac{1}{\varepsilon_{\infty}}-\frac{1}{\varepsilon}\right)\left(\frac{m_{b}}{2 \hbar \omega_{L O}}\right)^{1 / 2}
$$

em que $\varepsilon_{\infty}$ e $\varepsilon$ são as constantes dielétricas no limite de altas frequências e estática, respectivamente e $\Omega$ o volume do cristal polar. Um excelente artigo de revisão desse modelo do pólaron foi escrito pelo próprio Fröhlich.[13] As hipóteses assumidas na dedução do potencial de interação (Eq. 2) são: (a) o modelo continuum para o sólido é apropriado; b) a interação do elétron com fônons LO de grandes comprimentos de ondas é predominante; c) o espectro de banda é parabólico. Nesse caso, costuma-se designar a Hamiltoniana dada pela Eq. (1) como a do "pólaron grande", em que a extensão do pólaron é grande comparada com os parâmetros de rede, em oposição ao "pólaron pequeno", em que a aproximação de modelo continuum não é válida porque o elétron é localizado pelo campo de deslocamento atômico auto-induzido numa região da ordem do parâmetro de rede.[8] Convém notar que a constante de acoplamento é dada em termos de parâmetros acessíveis experimentalmente.

Fröhlich deduziu, a partir do efeito isotópico (a temperatura de transição do supercondutor depende do peso atômico do isótopo), que a interação elétron-fônon deve ser responsável pela supercondutividade. Em 1954, apre- 
sentou os resultados para a energia e massa efetiva dentro da teoria de perturbação de primeira-ordem (acoplamento fraco) [13]:

$$
\begin{aligned}
E_{0} & =-\alpha \hbar \omega_{L O} \\
m^{*} & =\frac{m_{b}}{1-\alpha / 6} .
\end{aligned}
$$

Esses resultados apresentados no seminário do Caltech mostravam uma divergência da massa efetiva para $\alpha$ da ordem de 6, o que poderia indicar uma transição de fase para um estado localizado do pólaron.

Na mesma época, Lee, Low e Pines (LLP) [14] usaram o formalismo de transformações canônicas e um tratamento variacional e encontraram $m^{*}=m_{b}\left(1+\frac{\alpha}{6}\right)$. Essa aproximação é conhecida como "teoria do acoplamento intermediário" e usada extensivamente em outras abordagens ao problema do pólaron como veremos a seguir. David Pines, na época pós-doc em Illinois, afirma [4] que foi levado pela intuição de John Bardeen de que a compreensão do problema do pólaron poderia ajudar no desenvolvimento de uma teoria microscópica da supercondutividade. Segundo Pines, Bardeen estava correto, uma vez que Schrieffer construiu para a Hamiltoniana de Bardeen, Cooper e Schrieffer (BCS) uma função de onda variacional adaptada da função de onda variacional do estado fundamental usada no tratamento de LLP.

O limite de acoplamento forte é obtido por meio da aproximação de Born-Oppenheimer considerando que o elétron segue adiabaticamente o movimento dos átomos e, portanto, que a função de onda do pólaron é o produto da função de onda do elétron (independente das coordenadas do campo de polarização) e do campo de fônons. O resultado nesse limite é

$$
\begin{aligned}
E_{0} & \left.=-\left(\alpha^{2} / 3 \pi\right) \hbar \omega_{L O}\right) \\
m^{*} & =m_{b}\left(1+0,02 \alpha^{4}\right) .
\end{aligned}
$$

Os resultados de acoplamento forte foram obtidos previamente por Landau e Pekar [11], mas não podiam ser aplicados haja vista os valores de $\alpha$ dos cristais polares.

Tornou-se evidente a necessidade de uma teoria do pólaron para todos acoplamentos.

No artigo seminal de 1955 [15], Feynman partiu da Hamiltoniana de Fröhlich (Eq. 1) e usou o método das integrais de caminho em "exact analogy to quantum electrodynamics" para determinar a energia e massa efetiva do pólaron no que é considerada ainda hoje a teoria válida para "todo acoplamento" e a mais bem sucedida abordagem a esse problema.

O procedimento usual consiste em resolver a função de onda do elétron que satisfaz $(\hbar=1)$

$$
i \frac{\partial \psi}{\partial t}=H \psi
$$

em que $H$ é dado pela Eq. (1). A solução geral da Eq. (6) pode ser escrita como

$$
\psi\left(\mathbf{r}^{\prime \prime}, t^{\prime \prime}\right)=\int d \mathbf{r}^{\prime} K\left(\mathbf{r}^{\prime \prime}, t^{\prime \prime} ; \mathbf{r}^{\prime}, t^{\prime}\right) \psi\left(\mathbf{r}^{\prime}, t^{\prime}\right)
$$

em que o propagador (função de Green) $K$ satisfaz a equação de Schrödinger para $t^{\prime \prime}>t$ ' com a condição inicial $\lim _{t^{\prime \prime} \rightarrow t^{\prime} \rightarrow 0} K\left(\mathbf{r}^{\prime \prime}, t^{\prime \prime} ; \mathbf{r}^{\prime}, t^{\prime}\right)=\delta\left(\mathbf{r}^{\prime \prime}-\mathbf{r}^{\prime}\right)$. Quando $H$ não depende explicitamente do tempo, as autofunções $\varphi_{n}$ e os autovalores $E_{n}$ de $H$ são dadas por

$$
\phi_{n}\left(\mathbf{r}^{\prime \prime}, t^{\prime \prime}\right)=\exp \left(-i E_{n} t^{\prime \prime}\right) \phi_{n}\left(\mathbf{r}^{\prime \prime}\right) .
$$

Expandindo $K$ nesses estados, obtém-se

$$
\begin{aligned}
& K\left(\mathbf{r}^{\prime \prime}, t^{\prime \prime} ; \mathbf{r}^{\prime}, t^{\prime}\right)=\left\langle\mathbf{r}^{\prime \prime}|\exp (i H t)| \mathbf{r}^{\prime}\right\rangle \\
& =\sum_{n} \exp \left[-i E_{n}\left(t^{\prime \prime}-t^{\prime}\right)\right] \phi_{n}\left(\mathbf{r}^{\prime \prime}\right) \phi_{n}\left(\mathbf{r}^{\prime}\right) .
\end{aligned}
$$

Mais interessante é trabalhar com a transformada de Fourier

$$
\begin{aligned}
& K\left(\mathbf{r}^{\prime \prime}, \mathbf{r}^{\prime} ; E\right)=-i \int_{0}^{\infty} \sum_{n} \exp \left[-i E\left(t^{\prime \prime}-t^{\prime}\right)\right] K \\
& \times\left(\mathbf{r}^{\prime \prime}, \mathbf{r}^{\prime} ; t^{\prime \prime}-t^{\prime}\right) d\left(t^{\prime \prime}-t^{\prime}\right),
\end{aligned}
$$

cuja expansão resulta em

$$
K\left(\mathbf{r}^{\prime \prime}, \mathbf{r}^{\prime} ; E\right)=\sum_{n} \frac{\phi_{n}\left(\mathbf{r}^{\prime \prime}\right) \phi_{n}\left(\mathbf{r}^{\prime}\right)}{E-E_{n}} .
$$

Considerando o plano complexo de $E$, como é bem conhecido, os polos de $K$ fornecem as energias e seus resíduos fornecem as funções de onda do sistema. Até aqui, nenhuma novidade. Trata-se de procedimento corriqueiro em problemas de uma partícula na mecânica quântica não relativística, reproduzido aqui por uma questão de completude. O interesse de Feynman é calcular o estado fundamental do pólaron, que pode ser difícil, usando Eq. (9) ou Eq. (11). Assim, considera fazer a substituição $T \equiv i\left(t^{\prime \prime}-t^{\prime \prime}\right) \rightarrow \infty$, tal que

$$
K\left(\mathbf{r}^{\prime \prime}, t^{\prime}-i T ; \mathbf{r}^{\prime}, t^{\prime}\right) \sim_{T \rightarrow \infty} e^{-E_{0} T},
$$

que corresponde à Eq. (8) do artigo original de Feynman. Assim, a taxa de decaimento do propagador fornece a energia do estado fundamental do pólaron.

O propagador é dado no formalismo da integral de caminho [1] por

$$
K=\int e^{S} D \mathbf{r}(\tau)
$$

em que a integral deve ser feita sobre todos os caminhos $\mathbf{r}(\tau)$ de $\exp (S)$. Transformando $H$, da Eq. (1), e a equação de Schrödinger, Eq. (6), na forma Lagrangeana da mecânica quântica e então eliminando o campo de fônons, chega-se a

$$
S=-\frac{1}{2} \int\left(\frac{d \mathbf{r}}{d \tau}\right)^{2} d \tau+\frac{\alpha}{2^{3 / 2}} \iint_{\tau^{\prime}}^{\tau^{\prime \prime}} d \tau d \sigma \frac{e^{-|\tau-\sigma|}}{|\mathbf{r}(\tau)-\mathbf{r}(\sigma)|}
$$


que é a Eq. (7) do artigo de Feynman. Essa ação é diferente da ação para tempos reais pelo fator $i$ (ver Eq. 4 do artigo).

O desafio agora é calcular a ação, dada pela Eq. (14), uma vez que apenas ações quadráticas possuem soluções analíticas. Feynman imaginou que deveria existir um princípio variacional "de algum tipo" que permitisse estimar integrais de caminho e chamou seu pós-doc Michel Baranger para lhe sugerir esse problema. Durante a conversa, Feynman tentou explicar o que deveria ser feito, enumerando todos os passos para se obter o tal princípio. Logo Baranger, para sua surpresa, percebeu que o problema sugerido já estava resolvido.

No depoimento a Jagdish Mehra [6], Feynman relembra:

I solved it in half an hour as I was explaining the problem. So I got excited and tried this variational method on the polaron problem. I discovered that with my variational method I could calculate the energy and also the mass of the polaron for all values of the coupling constant smoothly and continuously from one edge of the perturbation theory up to high energy, and at both ends it agreed with the best of others had done...It was really quite exciting, for I had discovered a variational principle at least for estimating path integrals, and it deepened my understanding. That turned out to be a powerful a discovered as the method of path integrals itself.

A citação acima demonstra a importância dada por Feynman à formulação de um princípio variacional para estimar integrais de caminho, já que apenas a ação quadrática tem solução analítica. Chamo atenção para a primeira frase do resumo do artigo original de 1955: "A variational principle is developed for the lowest energy of a system described by a path integral."

O método variacional de Feynman foi revisto em detalhes em sua palestra sobre o problema do pólaron em curso de 1961 [16]. O artigo original e as notas de aula de T.D. Schultz na Escola de Verão das Universidades Escocesas em 1962 servem de base para a discussão do método.[17]

Considere

$$
K=\int e^{S} D \mathbf{r}(\tau)=\int e^{S_{0}} e^{\left(S-S_{0}\right)} D \mathbf{r}(\tau)
$$

O lado direito pode significar o valor médio do funcional $\exp \left(S-S_{0}\right)$ sobre todos os caminhos $\mathbf{r}(\tau)$ com densidade de probabilidade $\exp \left(S_{0}\right)$, uma vez normalizada. Definindo para qualquer funcional $F[\mathbf{r}, \tau]$

$$
\langle F(\mathbf{r}, \tau)\rangle=\frac{\int e^{S_{0}} F(\mathbf{r}, \tau) D \mathbf{r}(\tau)}{\int e^{S_{0}} D \mathbf{r}(\tau)}
$$

e usando a desigualdade de Jensen $\left\langle e^{X}\right\rangle \geq e^{<X>}$, em que $X$ é uma variável aleatória, obtém-se

$$
\begin{aligned}
\int e^{S} D \mathbf{r}(\tau) & =\left\langle e^{S-S_{0}}\right\rangle \int e^{S_{0}} D \mathbf{r}(\tau) \\
& \geq e^{\left\langle S-S_{0}\right\rangle} \int e^{S_{0}} D \mathbf{r}(\tau)
\end{aligned}
$$

lembrando que para $T$ grande, foi mostrado que o estado fundamental do sistema é obtido por meio de

$$
\int e^{S} \operatorname{Dr}(\tau)=e^{-E_{g} T} .
$$

Se $S_{0}$ é uma ação tentativa, então, pela Eq. 18 pode-se escrever

$$
\int e^{S_{0}} \operatorname{Dr}(\tau)=e^{-E_{0} T}
$$

tal que para um sistema descrito por uma ação $S_{0}$, então $E_{0}$ será a energia do estado fundamental do sistema. Feynman propõe a seguir qual sistema seria esse. Desde que $S-S_{0}$, para $T$ grande, é proporcional a $T$, então

$$
\left\langle S-S_{0}\right\rangle=s T .
$$

Da Eq. 17) segue, então, o princípio variacional de Feynman

$$
E_{g} \leq E_{0}-\lim _{T \rightarrow \infty} \frac{1}{T}\left\langle S-S_{0}\right\rangle
$$

$\mathrm{Ou}$

$$
E_{g} \leq E_{0}-s .
$$

Feynman passa a examinar as possíveis escolhas das ações tentativas $S_{0}$. Sua "boa ideia" recai no uso da ação para uma partícula ligada a outra partícula via um potencial $V\left(\mathbf{r}-\mathbf{r}^{\prime}\right)$. O potencial escolhido é o harmônico, porque nesse caso a integração sobre a variável $\mathbf{r}^{\prime}$ pode ser feita e esta eliminada facilmente. Assim, Feynman introduz o exaustivamente usado modelo do pólaron concebido por um elétron ligado por meio de um potencial harmônico a uma partícula fictícia de massa finita que simula a essência da interação do elétron com a polarização da rede cristalina. A ação tentativa no modelo de Feynman é dada por

$$
\begin{aligned}
& S_{0}=-\frac{1}{2} \int\left(\frac{d \mathbf{r}}{d \tau}\right)^{2} d \tau-\frac{C}{2} \iint[\mathbf{r}(\tau)-\mathbf{r}(\sigma)]^{2} \\
& \times \exp (-w|\tau-\sigma|) d \tau d \sigma
\end{aligned}
$$

em que $C$ e $w$ são parâmetros escolhidos para minimizar o lado direito da Eq. (21).

O cálculo de $s$ da Eq. (20) não é trivial e Feynman detalha alguns truques no artigo, mas está mais bem elaborado na Ref. [17]. Os resultados para a energia no limite de acoplamento fraco ( $\alpha$ pequeno) são

$$
E_{g}=-\alpha-0,0123 \alpha^{2}+O\left(\alpha^{3}\right) .
$$

No limite de acoplamento forte $(\alpha \rightarrow \infty)$

$$
E_{g}=-\frac{\alpha^{2}}{3 \pi}-3 \ln 2-\frac{3}{4}+O\left(\frac{1}{\alpha^{2}}\right) .
$$


Como Feynman cita no resumo: "It is at least as accurate as previously known results".

O próximo passo consiste em determinar a massa efetiva do pólaron. Feynman lamenta que não conseguiu estender o princípio variacional que minimiza a energia para cada momento finito do elétron. Ele reconhece que o cálculo da massa efetiva não é rigoroso. Define a massa efetiva, admitindo que a auto energia do elétron deve ser acrescida de $E_{c}=m \times V^{2} / 2$ para velocidades pequenas. Considera o cálculo da ação sobre todos os caminhos desde a coordenada inicial $\mathbf{r}(0)=0$ até a final $\mathbf{r}(T)=\mathbf{U} T$ (U é uma velocidade imaginária). Assume que, para $T$ grande,

$$
\int_{\mathbf{r}^{\prime}}^{\mathbf{r}^{\prime \prime}} e^{S} D \mathbf{r}(\tau) \sim e^{-\left(E_{0}+\frac{1}{2} m^{*} U^{2}\right) T} .
$$

Os cálculos da massa efetiva estão detalhados na Ref. [17]. Os dois casos limites encontrados por Feynman são

$$
\begin{gathered}
m^{*}=1+\frac{\alpha}{6}+0,025 \alpha^{2}+\ldots \alpha \text { pequeno; } \\
m^{*}=\frac{16 \alpha^{4}}{81 \pi^{2}}=202\left(\frac{\alpha}{10}\right)^{4} \alpha \text { grande }
\end{gathered}
$$

em boa concordância com a teoria de acoplamento intermediário (LLP) e a teoria adiabática de acoplamento forte.

A tarefa de realizar os cálculos numéricos para todos os valores de $\alpha$ coube a Theodore Schultz (na época, doutorando no MIT) a pedido de Feynman. Ele conclui seu longo artigo com a sentença: "We have started with a comparison of the Feynman polaron theory with earlier theories of polaron self-energy and mass and have concluded that except in the truly weak and strong coupling situations it is the only satisfactory theory. [18]

A análise do problema do pólaron usando os já famosos diagramas de Feynman (19) foi feita logo a seguir em várias aproximações e para diferentes limites de acoplamento $\mathrm{O}$ resultado mais preciso em teoria de perturbação de mais alta ordem usando os diagramas mostrados na Fig. 2 para a energia do pólaron é dado por [20]

$$
\frac{E}{\omega_{0}}=-\alpha-1.591 .692\left(\frac{\alpha}{10}\right)^{2}-0,806\left(\frac{\alpha}{10}\right)^{3} \text {. }
$$

A teoria definitiva da mobilidade do pólaron baseada na formulação de integrais de caminh ${ }^{3}$ veio com o próprio Feynman ${ }^{4}$ e colaboradores [21] em 1962. A mobilidade $\mu$ é calculada a partir de impedância $Z(\omega)$ do pólaron

$$
\mu=\lim _{\omega \rightarrow 0} \operatorname{Re}[Z(\omega)]
$$

em que $\omega$ é a frequência do campo elétrico aplicado para valores arbitrários da constante de acoplamento e da temperatura. Uma abordagem baseada na matriz densidade foi desenvolvida em termos das integrais de caminho e o trabalho com cálculos bastante complexos foi revisitado por Platzman.[22]

A Fig. 3 apresenta a energia do pólaron como função do parâmetro de acoplamento $\alpha$ na aproximação de Feynman, obtida pela interpolação das expressões nos limites de acoplamento fraco e forte (Eqs. 23 e 24). A Fig. 3 mostra ainda, para comparação, dados da solução numérica, realizada por Schultz, e de outras aproximações. Como mostra a Fig. 3, a energia do pólaron apresenta comportamento suave em todo o intervalo de $\alpha$, de modo que o método de Feynman leva a uma transição contínua entre os regimes de acoplamento fraco e forte, eliminando a esperança de Fröhlich de que alguma singularidade essencial na interação elétron-fônon levasse a possível transição de fase supercondutora, como apontado pela divergência na massa efetiva para $\alpha$ da ordem de 6 , Eq. (4b).

Mas, Feynman tentou ainda responder por algum tempo à questão formulada a Fröhlich, "What do we have to do to understand superconductivity?" . No seu depoimento a Mehra [6] ele confessa sua enorme decepção em não ter conseguido entender o fenômeno:

The whole polaron problem had been a side issue from the beginning, as a sort of exercise and game, which gave me some pleasure. But it was only a side issue, not a central challenge. It's just that I happened to notice in the library that this problem was within the

\footnotetext{
${ }^{3}$ A primeira contribuição foi de Schultz em sua tese reportada na Ref. [18].

4 Segundo Mehra [6], Feynman havia sugerido a Schultz em maio de 1955 que seu formalismo de integrais de caminho deveria ser aplicado a "specific problems in which polarons are involved". E Schultz replicou que a mobilidade do polaron seria "a question of direct interest experimentally".
}
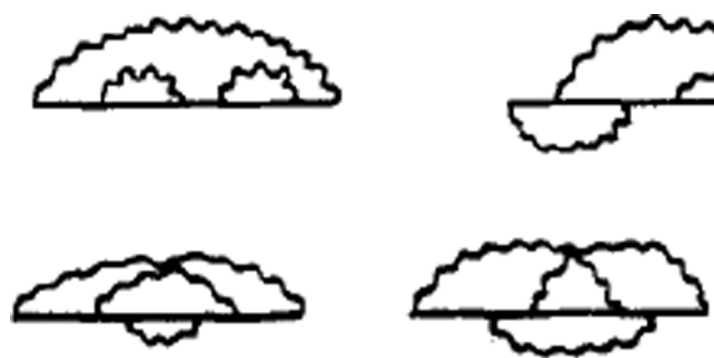
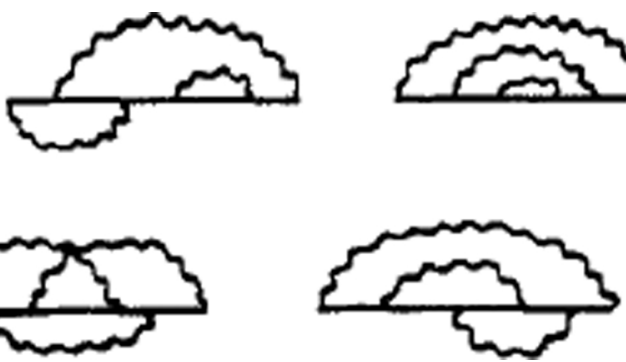
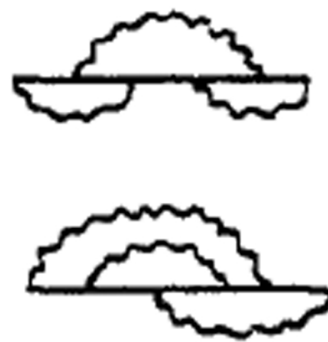

Figura 2: Diagramas de Feynman usados no cálculo da energia do pólaron em teoria de perturbação da $\mathrm{O}\left(\alpha^{4}\right)$. [20] 


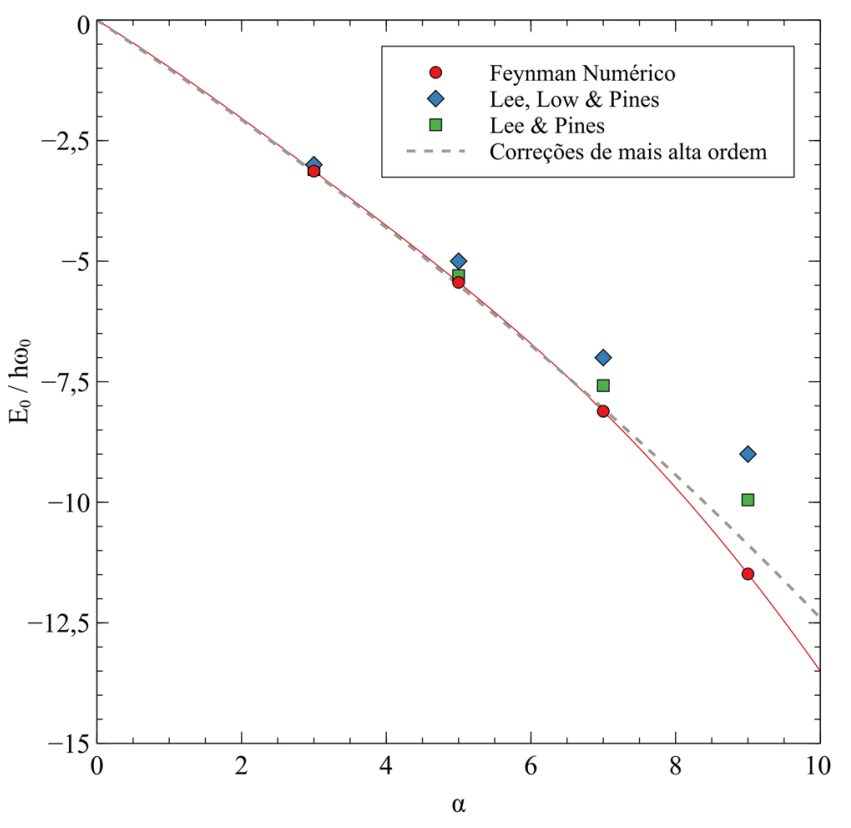

Figura 3: Energia do pólaron em função do parâmetro de acoplamento. A linha vermelha representa a interpolação dos resultados de Feynman no limite de acoplamento forte e fraco. As bolas vermelhas são os valores obtidos por Schultz resolvendo numericamente a integral de caminho. Os demais pontos são resultados das teorias de Lee-Low-Pines e Lee-Pines para comparação. Dados retirados da Ref. [18]. A linha tracejada corresponde ao resultado da teoria de perturbação da ordem de $\alpha^{4}$, realçando o mérito da aproximação de Feynman.

range of my tools, and I worked on it. For me, the central challenge was the problem of superconductivity, and I spent an awful lot of time in trying to understand it and doing everything by means of which I could approach it. I did an awful lot of calculations and developed a lot of methods, which I have gradually seen other people develop after me. But I did not solve the original problem. Where does superconductivity come from? I never published anything on it, and there is a big gap [in my publications] at that time, which was due to my attempts to solve the superconductivity problem - which I failed to do. I developed an emotional block against the problem of superconductivity, so that when I learned about the BCS paper I could not bring myself to read it for a long time. When I did read it, it was, of course, the right solution.

Tratando-se do caráter do Feynman, essa confissão de fracasso destoa de sua bem proclamada autoestima.

Após o trabalho de Feynman, logo seguiram-se várias aplicações da aproximação de Feynman para outros problemas que envolvem a interação do elétron com a rede: pólarons ligado a impurezas, pólarons de éxcitons, bipólarons, pólarons no campo magnético, entre outros.[17]

Como antecipado por Feynman em seu artigo, o método pode ser estendido facilmente para casos nos quais a frequência dos fônons não é constante e para outras formas de acoplamento do elétron com seu campo de polarização. Isto é, sempre que qualquer tipo de Lagrangeana quadrática possa modelar as características essenciais do sistema estudado.

O grupo liderado pelo Oscar Hipólito nos anos 70 e 80 em São Carlos, focava sua pesquisa, pioneira no Brasil, nos sistemas eletrônicos bidimensionais realizados em estruturas semicondutoras (camadas de inversão de MOSFETs, heterojunções e poços quânticos) e na superfície de hélio líquido.

Um elétron na superfície de um filme de hélio estabelece uma polarização no meio que pode ser calculada pelas leis da eletrostática. O resultado é o confinamento do elétron em um poço de potencial unidimensional na direção perpendicular à superfície devido a contribuições opostas: força atrativa devido à carga imagem atrativa, um campo elétrico externo $E_{\perp}$ que pressiona o elétron contra a superfície e uma barreira repulsiva devido ao princípio de exclusão de Pauli. No plano da superfície, os elétrons depositados se movimentam livremente (daí o caráter bidimensional) exceto pelo espalhamento por átomos do vapor de hélio e por ondas superficiais, espalhamento este dominante a temperaturas suficientemente baixas (menor que 0,5 K). Nesse caso a Hamiltoniana do sistema é do mesmo tipo da Hamiltoniana de Fröhlich com as excitações superficiais (ondas de capilaridade-gravidade), chamadas riplons (de ripples) na forma quantizada, fazendo o papel dos fônons. [23-26]

Seguindo a sugestão de Feynman de que seu método poderia ser aplicado a sistemas com frequência de fônons dependente do vetor de onda e generalizando para o caso de riplons, Hipólito, Farias e Studart [27] e independentemente Jackson e Platzman [28] calcularam a energia do estado fundamental e a massa efetiva do pólaron riplônico usando o formalismo de integral de caminho introduzido por Feynman para o problema do pólaron. No sistema elétron-riplon, o potencial de interação é dado por

$$
V_{k}=\left(\frac{k \tanh (k d)}{2 \varrho \omega_{k}}\right)^{1 / 2} e E_{\perp}
$$

e a lei de dispersão dos ripplons por

$$
\omega_{k}^{2}=g^{\prime} k \tanh (k d)\left[1+\frac{k^{2}}{k_{c}^{2}}\right]
$$

em que $g$ ' é aceleração gravitacional efetiva, $d$ a espessura do filme de hélio, $\rho$ a densidade do hélio e $k_{\mathrm{c}}$ a constante de capilaridade. Como no problema original, a energia do estado fundamental e a massa efetiva do pólaron riplônico variam uniformemente como função da espessura do filme e do campo elétrico externo. No entanto, no caso de filmes finos $(d \simeq 10 \mathrm{~nm})$ a dispersão de ripplons é típica 
de modo acústico $\left(\omega_{k}=s k\right) \operatorname{com} s=\left(g^{\prime} d\right)^{1 / 2}$, para $k<k_{c}$. Nesse caso especial, Jackson e Platzman [28] encontraram um comportamento para a massa efetiva do tipo de transição de fase de $1^{\mathrm{a}}$. ordem de um estado de elétron quase-livre para um estado auto-aprisionado. Esse resultado não surpreende porque na literatura encontram-se vários exemplos de auto-aprisionamento de um elétron pelo modo acústico da vibração da rede, desde o trabalho pioneiro de Toyozawa. [29] Os cálculos detalhados da energia e massa do pólaron riplônico estão na tese de doutorado de Gil Farias.[30] Degani e Hipólito usaram a aproximação de Lee-Low-Pines no estudo do estado polarônico no caso de único elétron e de muitos elétrons localizados em dimples sobre filmes de hélio líquido.[31]

Marques e Studart [32] realizaram um cálculo autoconsistente para determinar a mobilidade do pólaron no limite de acoplamento forte usando a aproximação adiabática proposta por Monarkha e Shikin [24] enquanto que Jackson e Peeters [33] seguiram o método de Feynman. O autor contribuiu, a convite, com artigo de revisão sobre o estado polarônico na superfície de filmes de hélio líquido que aborda inúmeros aspectos do problema, inclusive detalhando as contribuições do grupo de São Carlos.[34] O livro reúne contribuições de especialistas representativos dos diversos campos de estudo dos elétrons sobre hélio e outros substratos criogênicos. [26]

O caso acima é apenas um dos exemplos da aplicação do formalismo de integral de caminho ao pólaron e sistemas correlatos. Devreese e colaboradores na Antuérpia, com destaque para François Peeters, deram importantes contribuições ao desenvolvimento do estudo do pólaron, tanto à la Feynman, como em outras teorias. O conceito do pólaron foi estendido para os sistemas eletrônicos contemporâneos realizados em estruturas de dimensionalidade reduzida. No Brasil, o grupo de São Carlos realizou inúmeros estudos de pólarons em poços e fios quânticos de semicondutores.[35] Recomenda-se ao leitor interessado a leitura do livro de Alexandrov e Devreese $[36]$.

\section{Referências}

[1] J.D.M. Vianna, nessa seção especial da RBEF.

[2] V. Pleitez, nessa seção especial da RBEF.

[3] D. Kaiser, Drawing Theories Apart: The Dispersion of Feynman Diagrams in Postwar Physics (U. Chicago Press, Chicago, 2005).

[4] D. Pines, Physics Today 42, 2, 61 (1989).

[5] J. Leite Lopes, Ciência e Sociedade, Publicações do CBPF, no. 5 (1988).

[6] J. Mehra, The Beat of a Different Drum: The Life and Science of Richard Feynman (Clarendon Press, Oxford, 1994).

[7] P.F. Farinas, nessa seção especial da RBEF.

[8] J.T. Devreese, Encyclopedia of Applied Physics 14, 383 (1996). Disponível em https://arxiv.org/abs/condmat/0004497v2.
[9] L.D. Landau, Phys. Z. Sowjetunion 3, 664 (1933). Tradução para o inglês em Collected Papers of L.D. Landau, editado por D. Ter Haar (Pergamon Oxford, 1965), p. 67.

[10] M.I. Dykman and E.I. Rashba, Physics Today 68, 10 (2015).

[11] L.D. Landau and S.I. Pekar, Zh. Eksp. Teor. Fiz. 18, 419 (1948).

[12] H. Fröhlich, Proc. Roy. Soc. A 160, 230 (1937).

[13] H. Fröhlich, Advances in Physics 3, 325 (1954).

[14] T.D. Lee, F.E. Low and D. Pines, Phys. Rev. 90, 297 (1953).

[15] R.P. Feynman, Phys. Rev. B 97, 660 (1955).

[16] R.P. Feynman, Statistical Mechanics: A set of Lectures (Benjamin, Reading, 1972).

[17] T.D. Schultz, in: Polarons and Excitons, editado por C.G. Kuper and G.D Whitfield (Oliver and Boyd, Edimburg, 1963). p. 71.

[18] T.D. Schultz, Phys. Rev. 116, 526 (1959).

[19] A.C. Aguilar, nessa seção da RBEF.

[20] M.A. Smondyrev, Teor. Math. Fiz. 68, 29 (1986).

[21] R.P. Feynman, R.W. Hellwarth, C.K. Iddings and P.M. Platzman, Phys. Rev. 127, 1004 (1962).

[22] P.M. Platzman, in Polarons and Excitons, editado por C.G. Kuper and G.D Whitfield (Oliver and Boyd, Edimburg, 1963), p. 123.

[23] M.W. Cole, Rev. Mod. Phys. 46, 451 (1974).

[24] Yu. P. Monarkha and V.S. Shikin, Sov. J. Low Temp. Phys. 8, 279 (1982)

[25] N. Studart and O. Hipólito, Rev. Bras. Física 16, 194 (1986).

[26] E.Y. Andrei (ed), Two-Dimensional Electron Systems on Helium and Other Substrates (Kluwer Publ, Dordrecht, 1997).

[27] O. Hipólito, G.A. Farias and N. Studart, Surf. Sci. 113, 394 (1982).

[28] S.A. Jackson and P.M. Platzman, Phys. Rev. B 24, 499 (1981).

[29] Y. Toyozawa, Prog. Theo. Phys. 26, 29 (1959).

[30] G.A. Farias, Estados Superficiais em Sistemas Quase Bidimensionais. Tese de Doutorado, IFSC-USP (1980).

[31] M.H. Degani and O. Hipólito, Phys. Rev. 32, 3300 (1985); Surface Science 170, 97 (1986).

[32] G.E. Marques and N. Studart, Phys. Rev. B 39, 4133 (1989).

[33] S.A. Jackson and F.M. Peeters, Phys. Rev. B 30, 4196 (1984); F.M. Peeters and S.A. Jackson, ibid. 31, 7098 (1985).

[34] N. Studart and S.S. Sokolov, in Two-Dimensional Electron Systems on Helium and Other Substrates eitado por E.Y. Andrei (Kluwer Publ, Dordrecht, 1997).

[35] M.H. Degani and O. Hipólito, Phys. Rev. B 35, 4507, (1987).

[36] A.S. Alexandrov and J.T. Devreese, Advances in Polaron Physics, Springer Series in Solid-State Sciences, Vol. 159 (Springer-Verlag, Berlin, 2009). 